\title{
Type IV paraesophageal hiatal hernia and organoaxial gastric volvulus
}

\author{
Alison Zachry, Alan Liu, Sabiya Raja, Nadeem Maboud, Jyotu Sandhu, \\ DeAndrea Sims, Umer Feroze Malik*, Ahmed Mahmoud.
}

\begin{abstract}
Organoaxial gastric volvulus occurs when the stomach rotates on its longitudinal axis connecting the gastroesophageal junction to the pylorus. With that, the antrum of the stomach usually rotates in the opposite direction in relation to its fundus (1). This phenomenon has often been known to be associated with diaphragmatic defects (2) Importantly, abnormal rotation of the stomach of more than $180^{\circ}$ is a life threatening emergency that may create a closed loop obstruction which may result in incarceration leading to strangulation, and hence, a surgical emergency. We present the case of a middle-aged female who presented with organoaxial gastric volvulus and had an associated Type IV paraesophageal hiatal hernia that was treated electively. Normally an emergent gastric volvulus is diagnosed via Borchardt's classic triad (epigastric pain, unproductive vomiting and difficulty inserting a nasogastric tube); however in this patient the nasogastric tube (NGT) was passed into the antrum which allowed additional time for resuscitation with fluids and other symptomatic relief.
\end{abstract}

\section{CASE REPORT}

A 56 year old Caucasian female presented with inability to eat or drink due to persistent nausea and small amount of non bilious, non bloody emesis. The patient reported no bowel movements and an inability to pass flatus for one week. Patient had no other symptoms of intestinal obstruction and denied any urinary symptoms at that time.

Her past medical history included a chronic hiatus hernia of unknown etiology. The patient did not recall any further details regarding this hernia at the time. No previous images were found. Past surgical history included a laparoscopic cholecystectomy. She denied smoking, drinking alcohol, using recreational drugs, or having a relevant history. Aside from obstructive gastrointestinal symptoms,

To whom correspondence should be addressed: Umer Ferose Malik

Department of General Internal Medicine, Stanford University Medical Center (Welch Road), Stanford, Ca 94305, United States

Email: umalik@standford.edu a systemic review was negative. Abdominal examination revealed hyperactive bowel sounds. The patient's abdomen was mildly distended in the epigastric region. Her abdomen was soft with tenderness upon palpation in both the left upper quadrant and epigastric regions. There were no signs of an acute abdomen or peritonitis.

A complete laboratory workup revealed mild hypokalemia with no other significant findings. $A B G$ and lactate levels were not obtained at that time. Chest and abdominal radiographs showed an obvious stomach shadow in the thoracic area (Figures 2a and 2b). Computed tomography (CT) scans of the abdomen and pelvis showed a large hiatal hernia in the vertical sections (Figures 1a, 1b and 1c) however, it was even more apparent in the horizontal sections (Figures $3 a$ and $3 d$ ). Air-fluid levels were seen in the thoracic cavity extending left of the midline above the diaphragm at the level of T6 approximately. Fluid and a small amount of air was also visible in the abdominal cavity in the left 


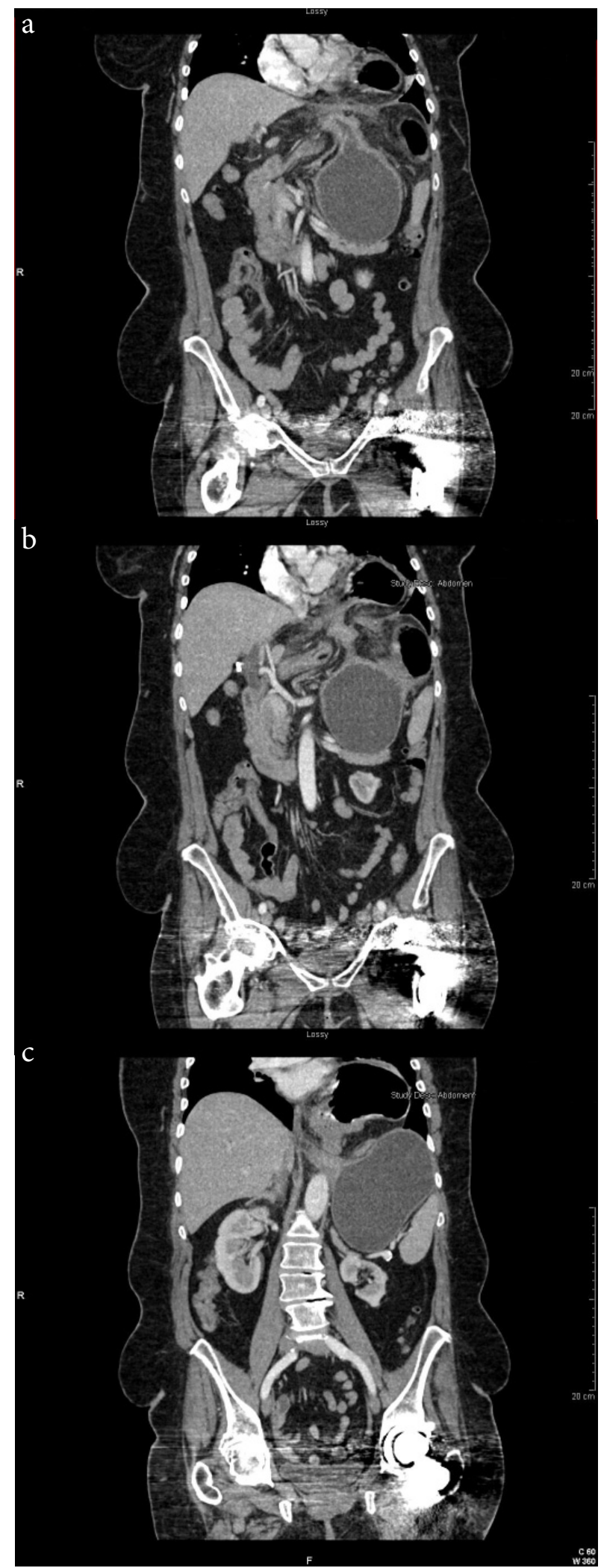

Figure 1 a) Computed tomography (CT) scan of the abdomen showing a large hiatal hernia in the vertical section.. b) Different view of Computed tomography (CT) scan of the abdomen and pelvis showing a large hiatal hernia in the vertical section. c) Computed tomography (CT) scans of the abdomen and pelvis with a large hiatal hernia in the vertical section. upper quadrant below the diaphragm. Furthermore, the antrum was identified in the thoracic cavity and the fundus was identified in the abdominal cavity. Organoaxial gastric volvulus was diagnosed radiographically as the stomach appeared twisted longitudinally.

Subsequently, the patient was admitted for close observation and further monitoring. An NGT was placed in the emergency department to decompress the stomach. When suctioned, the NGT removed $800 \mathrm{~mL}$ of clear gastric nonbilious fluid. An esophagogastroduodenoscopy (EGD) revealed scattered white exudation and circumferential inflammation of the esophageal mucosa, in which the severity was noted to be increased towards the distal end of the esophagus. Pressure at the lower esophageal sphincter was nearly absent. The distal gastric anatomy was distorted by the presence of a paraesophageal hernia. With slight resistance, the EGD passed into the antrum, which was noted to be entirely located within the thoracic cavity. Images of the antrum revealed diffusely granular and friable mucosa, and multiple small superficial mucosal erosions. During the EGD, the diseased mucosal margins were biopsied. Due to the marked anatomical distortions, the pylorus could not be identified. Pathology results showed chronic ulcerative gastritis with focal acute inflammatory changes. Biopsies were negative for Helicobacter pylori, dysplasia or malignancy.

After initiating conservative management and evaluating all treatment regimes, we concluded that the most necessary treatment was surgery. The patient was then taken to the operating room for reduction of the hiatal hernia and primary repair with gastropexy and pyloromyotomy of the incarcerated hiatal hernia. The reduction included much of the greater omentum in addition to part of the colon, which had also herniated into the thoracic cavity. The stomach was noted to be very large and floppy. The repair was successfully completed without complications. Following the procedure, complete relief of all gastrointestinal symptoms were achieved. Repeat CT scans confirmed resolution of the gastric volvulus and hiatal hernia (Figures 4a and 4c).

\section{DISCUSSION}

The very well known enigma, gastric volvulus, was first described by Berti in 1866 while performing a postmortem exploration (3). In 1896, Berg performed the first successful operation on a patient with gastric volvulus (4). In 1904, Borchardt described the classic triad of this condition: severe 

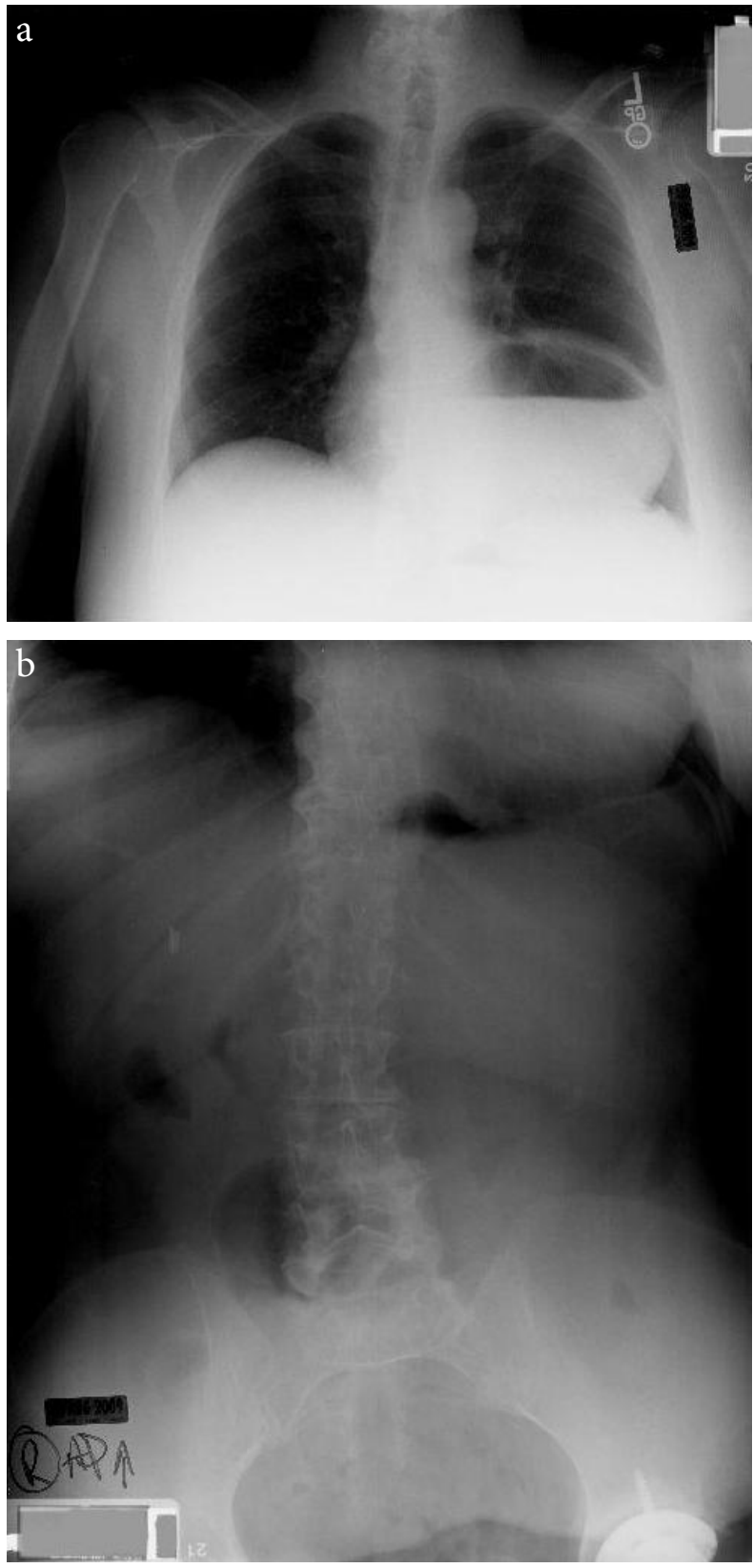

Figure 2. a) Chest x-ray showing stomach shadow in the thoracic area. b) Chest and abdominal radiograph revealing stomach shadow in the thoracic area.

epigastric pain, retching without vomiting, and the inability to pass an NGT (5). With technical advancements, surgeons have described this entity in various settings enabling physicians to more efficiently and effectively manage patients with this condition.

On occasion, a gastric volvulus may form as a complication of a hiatal hernia. This most commonly presents with the stomach displaced through the esophageal hiatus of the diaphragm into the thoracic region. Three different types of gastric volvuli exist: organoaxial, mesenteroaxial, or a combined volvulus. There are four different types of hiatal hernias that may result in a gastric volvulus: sliding, paraesophageal, combination of sliding and paraesophageal, or complex paraesophageal hiatal hernia.

Type I, also known as sliding hiatal hernia, is the most common type and usually associated with gastroesophageal reflux disease (GERD). The stomach, or part of it, usually slides in and out of the hernia. When a part of the stomach squeezes through the hiatus into the thoracic cavity, adjacent to the esophagus, it is called a Type II or paraesophageal hiatal hernia. A combination of sliding and paraesophageal hiatal hernias is categorized as Type III. If additional abdominal contents are found in the thoracic cavity then a diagnosis of Type IV or complex paraesophageal hiatal hernia is given. A Type IV hernia may include the whole stomach, the small and large bowels, spleen, pancreas, or liver (6).

A patient may present with one of the four types of hiatal hernias in addition to having a gastric volvulus. Organoaxial gastric volvulus occurs when the stomach rotates on its longitudinal axis connecting the gastroesophageal junction to the pylorus. With that, the antrum of the stomach usually rotates in the opposite direction in relation to its fundus $(1,7)$. It is the most common type of gastric volvulus, making up $59 \%$ of all cases (8). In a mesenteroaxial volvulus, the stomach rotates around the gastrohepatic omentum in a left/right or right/left direction (9). The mesoaxial gastric volvulus makes up $29 \%$ of all cases of gastric volvuli (8). The combined form is a rare occurrence in which the stomach twists in a mesentericoaxial and organoaxial fashion. Regardless, in all types, the patient may become symptomatic from vascular compromise or gastric outlet obstruction and present with the classic Borchardt's triad. With vascular compromise the mortality rate is nearly $30 \%$, making a gastric volvulus an emergent diagnosis (10). In this case, the patient suffered a type IV paraesophageal hiatal hernia with organoaxial gastric volvulus. With emergent diagnosis, gastric ischemia was prevented.

The paraesophageal hernias, as seen in our patient, tend to enlarge overtime, increasing the risk of gastric volvulus. Once this occurs, there is an even greater risk of incarceration, possible strangulation, or even perforation. Surgical management is indicated in roughly $5 \%$ of paraesophageal hernia cases (11). 

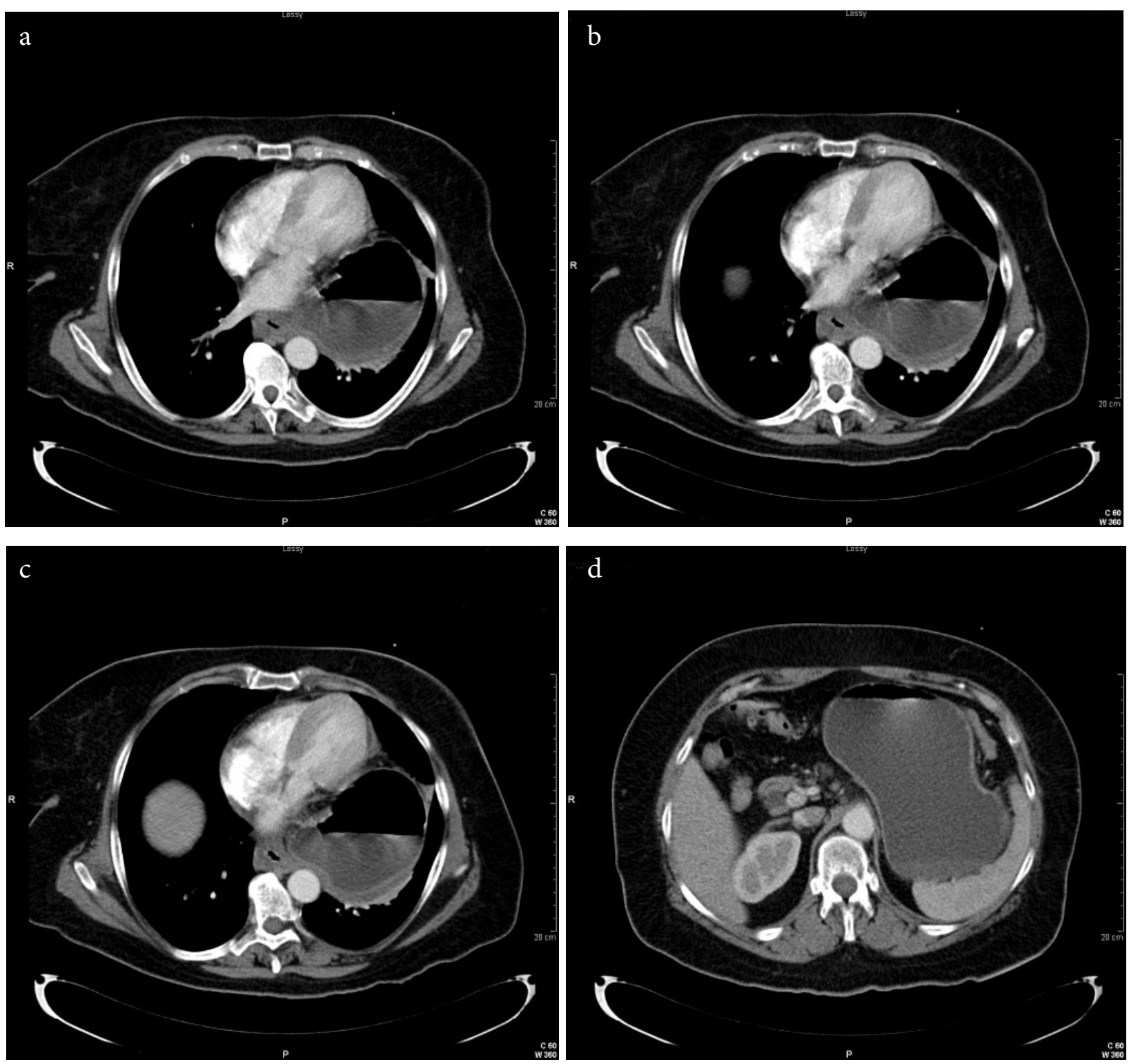

Figure 3: a) Horizontal section of the Computed tomography (CT) scan of the chest showing a large hiatal hernia b) Computed tomography (CT) scans of the chest showing a large hiatal hernia, a horizontal view. c) Computed tomography (CT) scans of the chest and abdomen showing a large hiatal hernia (Horizontal view). d) Horizontal Computed tomography (CT) scan of the abdominal area showing a hiatal hernia.

Due to the possibility of mortality with hiatal hernias, it is important for clinicians to recognize the various factors that may contribute to their development. These include: trauma, an inherent weaknesses in the surrounding muscles, straining during heavy weight lifting, having an unusually large hiatus from birth, or persistently intense pressure on the surrounding muscles, which may occur with vomiting or straining during a bowel movement. Additional risk factors include age (>50 years), obesity, pregnancy, and smoking (12).

Patients with hiatal hernias vary widely in their experience of symptoms. They are usually related to the development of GERD, which may often occur before or after the development of the hernia. These symptoms may include heartburn, gastric discomfort, chest pain, increased belching, hoarseness, throat irritation, dysphagia, haematemesis or malaena. However, it is also very common for patients to be completely asymptomatic. As in the case presented above, it is worth noting that the severity of symptoms does not necessarily correlate with the severity of the hernia.

A diagnosis of a hiatal hernia and gastric volvulus may be suspected based on clinical presentation, however, a thorough workup 

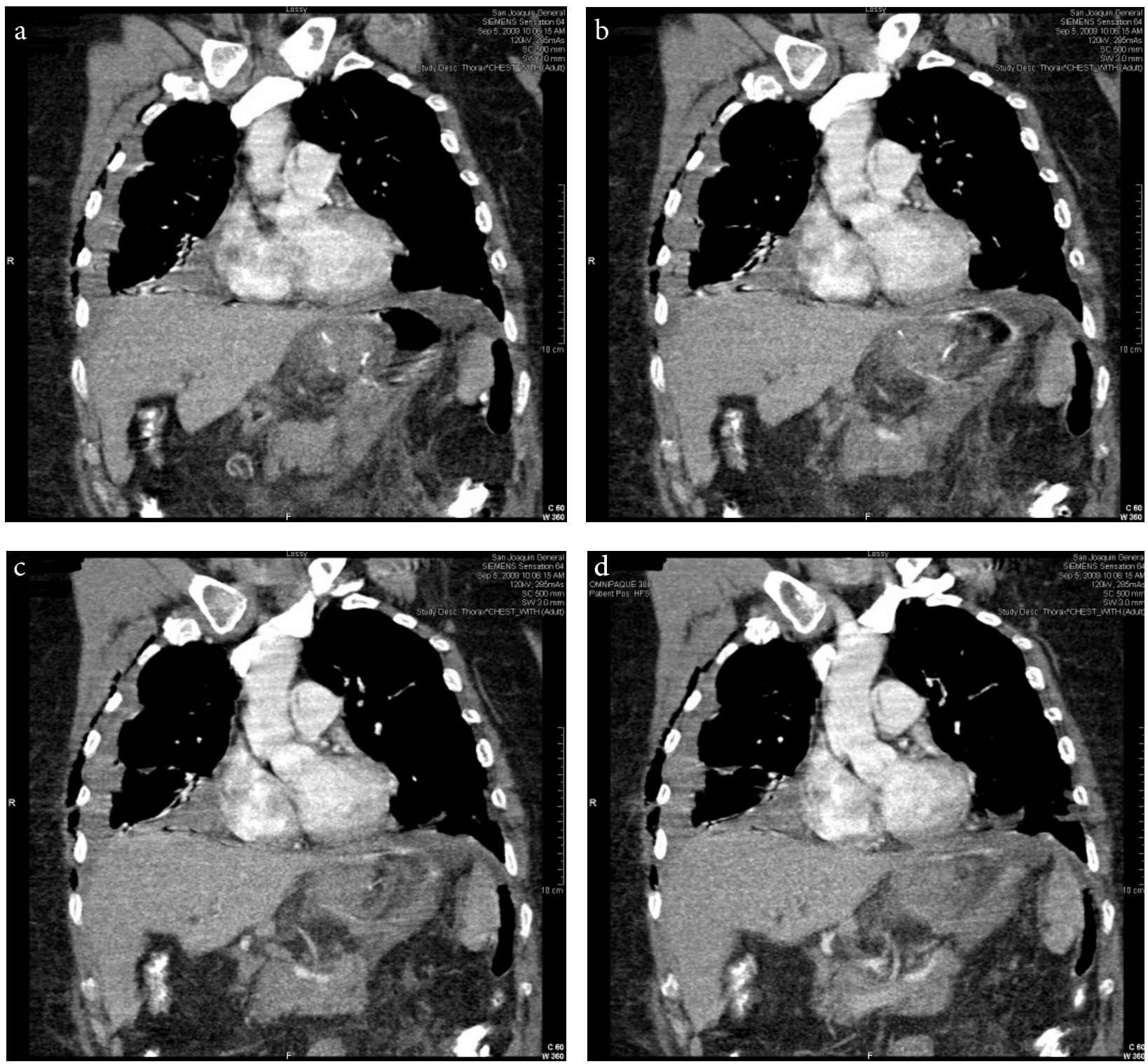

Figure 4: a) Thoraco-abdominal vertical view of the CT scan obtained after the surgical treatment confirmed resolution of the gastric volvulus and hiatal hernia. b) Thoraco-abdominal vertical view of the CT scan confirmed resolution of the gastric volvulus and hiatal hernia after the procedure. Post surgical changes are also apparent. c) Thoraco-abdominal vertical view of the CT scan confirmed resolution of the gastric volvulus and hiatal hernia after the procedure. Stomach is apparent in the abdominal cavity after primary gastropexy. d) Complete esolution of the gastric volvulus and hiatal hernia is shown in the CT scan, vertical view. Signs of primary repair with gastropexy and pyloromyotomy are also apparent upon close evaluation.

including imaging studies of the chest and abdomen with barium upper gastrointestinal series should be ordered. Radiological signs of gastric volvulus include a double air-fluid level on upright films, inversion of the stomach with the greater curvature above the level of the lesser curvature, positioning of the cardia and pylorus at the same level, and downward pointing of the pylorus and the duodenum (13). In addition, chest radiography may reveal a retrocardiac air-filled mass and abdominal films may reveal an increased soft tissue density in the upper abdomen consistent with a distended fluid filled stomach (2). The barium studies are both sensitive and specific and can confirm a diagnosis (2). It is important to rule out different etiologies including unknown masses and malignancies, which may be confirmed with endoscopic biopsy. Treatment depends on the severity of the hernia and can range from lifestyle changes to surgical intervention as seen in our patient.

Patients diagnosed with hiatal hernias should first be counseled on the importance of lifestyle changes to manage their condition. This includes a high fiber diet to decrease the amount 
of straining during bowel movements and avoiding foods such as alcohol, chocolate, citric juice, and tomato-based products as these may decrease pyloric sphincter tone. Patients should also avoid eating large meals and eating too quickly as this stretches the stomach and relaxes the lower esophageal sphincter. Moderate exercise should be encouraged to maintain a healthy body mass index (BMI). Medications are also helpful in managing this condition and may include antacids, $\mathrm{H}-2$ receptor blocking drugs or proton pump inhibitors. Prokinetic agents are also warranted in patients with mild symptoms but long term use is discouraged due to their potentially fatal complications. In some cases, endoscopic repair may be warranted before surgical intervention is necessary (14).

A minority of patients may require surgery. These patients have usually failed to improve even after aggressive proton pump inhibitor treatment and lifestyle modifications. Other groups may be those suffering from pulmonary complications such as asthma, aspiration pneumonia, chronic cough or hoarseness related to reflux disease. If the patient is found to have a gastric volvulus, surgical intervention is highly indicated as the mortality rate without it has been reported to be as high as $80 \%(15,16,17,18)$. In these cases, three types of surgical approaches may be performed. These include Nissen Fundoplication, Belsey (Mark IV) Fundoplication and Hill repair approach (19).

Our patient suffered a type IV paraesophageal hiatal hernia with organoaxial gastric volvulus. A unique discovery in this case was our ability to advance the NGT (20) which allowed more time for additional studies, thus sparing the patient from an emergent invasive surgical procedure. With additional time, we were able to adequately resuscitate the patient and provide comfort measures as well as optimizing the patient for surgery.

\section{ABBREVIATIONS}

ED-Emergency Department, CT-computer tomography, BMI-Body Mass Index, GERDgastroesophageal reflux disease, NGT-nasogastric tube, EGD-esophagogastroduodenoscopy.

\section{CONFLICTS OF INTEREST}

The authors declare that they have no conflicts of interest

\section{CONSENT}

Written informed consent was obtained from the patient for publication of this case report and accompanying images.

\section{REFERENCES}

1. Wasselle JA, Norman J. Acute gastric volvulus: Pathogenesis, diagnosis, and treatment. Am J Gastroenterol. 1993;88:1780-1784.

2. McElreath DP, Olden KW, Aduli F. Hiccups: A Subtle Sign in the Clinical Diagnosis of Gastric Volvulus and a Review of the Literature. Digestive Diseases and Sciences. 2008; 53(11):3033-3036.

3. Berti A. singulare attortigliamento dele' esofago col duodeno seguita da rapida morte. Gazz Med Ital. 1866;9:139.

4. Berg J. Zwei Falle von axendrehung des magens operation;heilung. Nord Med Arkiv. 1897;30:1.

5. Borchardt M. Aus Pathologie und therapie des magenvolvulus. Arch Klin Chir. 1904;74:243.

6. Kahrilas PJ, Kim HC, Padnolfino JE. Approaches to the diagnosis and Grading of Hiatal Hernia. Best Practive and Research: Clinical Gastroenterology. 2008; 22(4): 601616.

7. Sevcik WE, Steiner IP. Acute gastric volvulus: A case report \& review of literature CJEM, 1999; 3: 200-203

8. Milne LW, Hunter JJ, Anshus JS. Gastric volvulus: two cases and a review of the literature. J Emerg Med. 1994;12(3):299-306.

9. Campbell JB, Rappaport LN, Skerker LB. Acute mesentero-axial volvulus of the stomach. Radiology. 1972;103:153-156.

10. Carter R, Brewer LA 3rd, Hinshaw DB. Acute gastric volvulus. A study of 25 cases. Am J Surg. 1980;140:99106.

11. Sihvo El, Salo JA, Rasanen JV, Rantanen TK. Fatal complications of adult paraesophageal hernia: a population-based study. J Thorac Cardiovasc Surg. 2009;137(2):419-424.

12. Shivanand G, Seema Seemar, Srivastava DN, Pande GK, Sahni P. Gastric volvulus: Acute and chronic presentation. Clinical Imaging. 2003;27(4): 265-268.

13. Eisenberg RL. Gastric volvulus. In: Gastrointestinal Radiology.A Pattern Approach. Philadelphia, Pa: JB Lippincott; 1983:286-288.

14. Hisatsune $\mathrm{H}$, Yohsida $\mathrm{S}$, Mizuno $\mathrm{S}$, Oki $\mathrm{T}$, Mukai $\mathrm{M}$, Marui K, Hirayama T, Itoh T, Kimoto K. A case of Acute organoaxial gastric volvulus treated by endoscopic reduction and fixation with the use of percutaneous endoscopic gastrostomy devia. Digestive Endoscopy Journal for Gastroenterologists and Endoscopic Surgeons. 2007:8(4);324 - 327.

15. Aye RW, Hill LD, Kraemer SJ, Snopkowski P. Early results with the laparoscopic Hill repair. American Journal of Surgery. 1994;167(5):542-6.

16. Migliore M, Arcerito M, Vagliasindi A, Puleo R, Basile F, Deodato G. The place of Belsey Mark IV fundoplication in the era of laparoscopic surgery. European Journal of Cardiothoracic Surgery. 2003;24(4):625-30.

17. Palanivelu C, Rangarajan M, Shetty AR, et al. Laparascopic suture gastropexy for gastric volvulus: a report of 14 cases. Surg Endosc. 2007;21(6):863-6.

18. Papasavas PK, Keenan RJ, Yeaney WW, Caushaj PF, Gagne DJ, Landreneau RJ. Effectiveness of laparascopic fundoplication in relieving the symptoms of gastroesophageal reflux disease (GERD) and eliminating antireflux medical therapy. Surg Endosc. 2003;17(8):1200-5.

19. DiMarino Jr A, Benjamin S (2002). Gastrointestinal Disease: An Endoscopic Approach (2nd ed.). Thorofare, NJ: Slack Incorporated.

20. Moy R, Salazar A, Chan S. Inability to pass a nasogastric tube: a surgical emergency. The American Journal of Emergency Medicine. 2007;25(2):213-215. 